\title{
Principios de estadística Bayesiana y su relación con la farmacocinética aplicada
}

\section{Principles of Bayesian statistics and its relationship with applied pharmacokinetics}

\author{
Paulo Cáceres Guido ${ }^{a}$, Carlos Humberto Pavan ${ }^{\mathrm{b}}$, Esteban Otamendi ${ }^{\mathrm{c}}$, Guillermo Federico Bramuglia ${ }^{\mathrm{d}}$
}

\author{
aUnidad de Farmacocinética Clínica (Farmacia), Hospital de Pediatría Prof. Dr. Juan P. Garrahan. Argentina \\ baboratorio Nacional de Investigación y Servicios en Péptidos y Proteínas. Estudios por Espectrometría de Masas. LANAIS-PROEM. \\ Universidad de Buenos Aires - CONICET. Argentina \\ Laboratorio de Urgencias. Hospital Donación Francisco Santojanni. Argentina \\ ¿Cátedra de Farmacología. Facultad de Farmacia y Bioquímica. Universidad de Buenos Aires. Argentina
}

Recibido: 06 de enero de 2020; Aceptado: 17 de mayo de 2020

\section{¿Qué se sabe del tema que trata este estudio?}

La Farmacocinética ayuda a conocer características fundamentales de los fármacos. En la rutina clínica, suele desconocerse el comportamiento farmacocinético de un paciente individual. La implementación de ciertos principios estadísticos sustenta la elección de una posología para optimizar la farmacoterapia.

\begin{abstract}
¿Qué aporta este estudio a lo ya conocido?
La estadística Bayesiana, basándose parcialmente en características de la población a la que un paciente pertenece, permite estimar parámetros farmacocinéticos de una droga en ese paciente. Este manuscrito explica los fundamentos que hacen posible optimizar más eficientemente regímenes farmacoterapéuticos individuales.
\end{abstract}

\section{Resumen}

La metodología estadística Bayesiana permite, si se conoce la probabilidad poblacional de que un suceso ocurra, modificar su valor cuando se dispone de nueva información individual. Aunque las metodologías Bayesiana y frecuentista (clásica) tienen idénticos campos de aplicación, la primera se aplica cada vez más en investigación científica y análisis de big data. En la farmacoterapia moderna, la farmacocinética clínica ha sido responsable de la expansión de la monitorización, facilitada por desarrollos técnico-analíticos y matemático-estadísticos. La farmacocinética poblacional ha permitido identificar y cuantificar las características fisiopatológicas y de tratamiento en una población de pacientes determinada, en particular en pediatría y neonatología, y otros grupos vulnerables, explicando la variabilidad farmacocinética interindividual. Asimismo, la estimación Bayesiana resulta importante como herramienta estadística aplicada en programas informáticos de optimización farmacoterapéutica cuando la monitorización farmacológica se basa en la interpretación farmacocinética clínica. Aunque con ventajas y limitaciones, la optimización farmacoterapéutica basada en la estimación Bayesiana es cada vez más usada en la actualidad, siendo el método de referencia. Esto es
Palabras clave:

Farmacocinética;

Estadística \& Datos

Numéricos;

Optimización;

Farmacología;

Teorema de Bayes

Correspondencia:

Paulo Cáceres Guido

caceresguido@gmail.com 
particularmente conveniente para la práctica clínica de rutina debido al limitado número de muestras requeridas por parte del paciente, y a la flexibilidad en cuanto a los tiempos de muestreo de sangre para cuantificación de fármacos. Así, la aplicación de los principios Bayesianos a la práctica de la farmacocinética clínica resulta en la mejora de la atención farmacoterapéutica.

\section{Keywords:}

Pharmacokinetics; Statistics and Numerical Data; Optimization; Pharmacology; Bayer Theorem

\section{Introducción}

Hasta principios del siglo XX, los investigadores sanitarios difícilmente dominaban métodos para cuantificar la "evidencia” y complementar informes "anecdóticos" de sus investigaciones ${ }^{1,2}$. Desde entonces se desarrollaron las "Pruebas de Hipótesis", procedimientos basados en evidencia muestral (estadístico o medida cuantitativa derivada de un conjunto de datos de una muestra) y en la Teoría de la Probabilidad (distribución del estadístico en la muestra) para aceptar o rechazar la hipótesis de investigación, basadas en calcular los Valores de Probabilidad (valor de P), y conocidas como Pruebas de Significación Estadística (PSE) ${ }^{1}$.

La estadística frecuentista, desarrollada a partir de los conceptos de probabilidad y centrada en el cálculo de probabilidades y en los contrastes de hipótesis, es en la que mayormente se enfocan los sistemas educativos actuales. Por ello, los principios de la estimación Bayesiana, aun sin ser de extrema complejidad, podrán todavía resultar relativamente novedosos ${ }^{1,3-5}$.

En el campo clínico, la aplicación de un tratamiento u otro, o la elección, para un mismo fármaco, de una posología por sobre otra, están estrechamente ligadas a las conclusiones a las que se arriba luego de la aplicación de los procedimientos estadísticos antes mencionados. Por ello, un conocimiento más profundo de los fundamentos de los mismos posibilita actuar con mayor solidez profesional, tanto a la hora de analizar fuentes de información científica como al momento de tomar la mejor decisión terapéutica.

\section{Teoría de la Probabilidad y Estadística Frecuentista}

La Teoría de la Probabilidad se basa en principios fundamentales e indemostrables llamados axiomas que son resultado de décadas de evolución, y que se concretó definitivamente hacia la segunda mitad del siglo XVII, con los trabajos matemáticos de Blaise Pascal y Pierre de Fermat ${ }^{6}$. Recientemente, el creciente acceso a potentes recursos computadorizados, así como también el desarrollo de numerosos paquetes estadísticos, tales como $\mathrm{SPSS}^{\circledR}, \mathrm{SAS}^{\circledR}, \mathrm{STATA}^{\circledR}, \mathrm{EPINFO}^{\circledR}$, e incluso EXCEL $^{\circledR}$, hicieron que el uso de las PSE ganara espacio en la investigación biomédica ${ }^{7,8}$. Considerando que la Hipótesis Nula $\left(\mathrm{H}_{0}\right)$ es lo que el investigador sospecha que no es cierto (es decir, lo que se quiere probar), las PSE permiten establecer que tan probable es encontrar una diferencia entre distintos tratamientos si la $\mathrm{H}_{0}$ es cierta. Las PSE ponderan qué tan probable es encontrar la diferencia que se detectó, por ejemplo, entre dos tratamientos analizados. Esta "Probabilidad" es el valor P, y se asocia al concepto de "significación (o significancia) estadística" ${ }^{1,5}$.

La aparente simplicidad conceptual del valor de "P" lo hace atractivo y confiable en el ámbito clínico, 
ya que su valor depende de la magnitud de la diferencia que queremos probar, así como del tamaño muestral $^{5}$.

Algunas objeciones al uso de las PSE son ${ }^{1}$ :

a) Los valores de P no cuantifican la probabilidad de que, a la luz de los datos, una hipótesis determinada sea cierta, que es lo que realmente interesa, sino la probabilidad de haber obtenido ciertos datos en el supuesto de que sea cierta determinada hipótesis;

b) El rechazo de $\mathrm{H}_{0}$ depende del tamaño muestral, que es un elemento algo ajeno a la realidad;

c) Las PSE se basan en un esquema que es dicotómico (clasifica las experiencias según solo dos categorías opuestas, sin posibilidad de situaciones intermedias) y mecanicista (concibe todos los hechos como producto de procesos físicos y químicos), por lo que no permite mayores deducciones directas, siendo estas necesarias para que la realidad estudiada sea mejor entendida;

d) Las PSE trabajan en un ámbito en el que no es necesaria la conexión entre pruebas y conocimientos previos;

e) Para valorar la magnitud de $P$ se emplean umbrales $(0,05$ o 0,01$)$ que carecen de fundamento matemático suficientemente sólido.

Una alternativa a las PSE, en una investigación, es construir un Intervalo de Confianza (IC). El IC, aludiendo a la firme esperanza (presunción) sobre algo, describe la variabilidad entre la medida obtenida en un estudio y la medida verdadera (real) de la población. Es un rango de valores (calculado de una muestra con distribución normal) en el cual se encuentra el verdadero valor del parámetro, con una probabilidad determinada ${ }^{1,9}$.

La probabilidad de que el verdadero valor del parámetro se encuentre en el intervalo construido se denomina Nivel de Confianza, y se denota " $1-\alpha$ ", donde " $\alpha$ " es la probabilidad de cometer error de tipo I (error falso positivo $=$ probabilidad de rechazar la $\mathrm{H}_{0}$ siendo que esta es positiva). Generalmente se construyen intervalos con confianza $1-\alpha=95 \%$ (o significancia $=5 \%)$, o menores $(\alpha=1 \%)^{9}$.

Los IC y las PSE están condicionados por el tamaño muestral. Una muestra suficientemente grande ocasionalmente podrá lograr un intervalo lo suficientemente estrecho como para que el 0 , si se tratara por ejemplo de una diferencia, quede fuera del intervalo, y así se rechace la hipótesis de que los grupos comparados son iguales ${ }^{1,9}$.

La probabilidad, puede tener dos significados diferentes. Por un lado, la estabilización de la frecuencia de aparición de un fenómeno y por otro una medida de certeza de la veracidad de una proposición. A la prime- ra se la conoce como probabilidad frecuentista (clásica), a la segunda como Bayesiana ${ }^{3,4}$.

La interpretación frecuentista del concepto de probabilidad considera a esta última como la frecuencia relativa de un experimento aleatorio. Sin embargo, el enfoque Bayesiano interpreta la probabilidad de una manera algo más subjetiva, condicionándola parcialmente a cierta evidencia parcial previa. El método Bayesiano hace uso de la información contenida en relación a un paciente, pero no depende solo de esta al momento de la toma de una decisión. En términos coloquiales, y considerando ambos enfoques en la práctica clínica, la estimación Bayesiana siempre resulta algo más “intuitiva”. En términos descriptivos, una aproximación Bayesiana proporcionaría una conclusión como la siguiente: "hay un 95\% de probabilidad de que el valor esté entre 0,7 y 0,9 ”, y por otro lado, los principios frecuentistas tenderían a dar explicaciones como la siguiente: "si generáramos 100 muestras aleatorias del mismo tamaño y repitiéramos la estimación 100 veces, en 95 de ellas el parámetro estimado estaría entre 0,7 y $0,99^{\prime 2,4,5}$.

\section{Comienzos y Fundamentos de la Estadística Bayesiana}

El matemático y ministro presbiteriano británico Thomas Bayes expuso sus teorías sobre la probabilidad en forma póstuma, en 1763: "An Essay towards solving a Problem in the Doctrine of chances" (Philosophical Transaction of the Royal Society), dividido en dos secciones ${ }^{10}$. En la primera incorpora definiciones, teoremas y corolarios sobre la probabilidad, y en la segunda descubre un modelo que le permite expresar y demostrar su teorema. Se le considera el fundador de la probabilidad inversa, según la cual, a partir de cierta información muestral es posible conocer cuan probable es que un suceso desconocido esté comprendido entre ciertos límites. Así, con Bayes, comienza la inferencia estadística y una interpretación diferente del concepto de probabilidad, constituyendo una alternativa a la estadística frecuentista (clásica o tradicional) ${ }^{3,4}$.

En el campo frecuentista se calcula la probabilidad de observar un resultado suponiendo que la realidad es de una manera determinada $\left(\mathrm{H}_{0}\right)$. Acorde a esto, cuantas más veces se repita el experimento, finalmente la posibilidad de que ocurra cada uno de los sucesos será conocida y regular. En la práctica necesitamos conocimientos para tomar decisiones, y lo que realmente nos interesa es conocer la probabilidad de que las cosas sean de una manera determinada dados los datos que hemos observado. La estadística Bayesiana, a diferencia de la frecuentista, incorpora información externa al estudio para, con ella y los propios datos observados, 
estimar una distribución de probabilidad para la magnitud del efecto que se investiga ${ }^{3,4,11}$.

\section{Metodología Bayesiana}

El método Bayesiano permite, si se conoce la probabilidad de que ocurra un suceso, modificar su valor cuando se dispone de nueva información ${ }^{5,12,13}$.

Aunque para el ámbito clínico pueda resultar algo tedioso conocer la base bioestadística que subyace al pensamiento Bayesiano, vale hacer un pequeño esfuerzo, ya que una rápida aproximación será suficiente para una comprensión suficientemente profunda del mismo.

Siendo ${ }^{5,12,13}$

- $\quad$ P(A) la probabilidad de que ocurra el suceso A.

- $\quad$ P(A.B) la probabilidad de que ocurran los sucesos A y B (ambos).

- $\quad \mathrm{P}(\mathrm{A} / \mathrm{B})$ la probabilidad de que ocurra A cuando sabemos que ha ocurrido B (se denomina probabilidad condicionada).

La probabilidad de que ocurra A y B es igual a la probabilidad de B multiplicada por la probabilidad de $A$ condicionada a que haya ocurrido $B$.

$\mathrm{P}(\mathrm{A} . \mathrm{B})=\mathrm{P}(\mathrm{B}) \times \mathrm{P}(\mathrm{A} / \mathrm{B})=\mathrm{P}(\mathrm{A}) \times \mathrm{P}(\mathrm{B} / \mathrm{A})$

Por simetría se cumple la tercera igualdad.

Si hay un conjunto de posibles sucesos mutuamente excluyentes $A_{i}(i=1 \ldots n)$ que constituyen todas las posibles situaciones (o lo que es lo mismo $\mathrm{P}(\mathrm{A} 1)+\mathrm{P}(\mathrm{A} 2)+\ldots+\mathrm{P}(\mathrm{An})=1)$, el que ocurra alguno de los sucesos A tiene probabilidad 1 (Figura 1). El cuadrado corresponde a todas las situaciones posibles, que en este caso pueden dividirse en tres: A1, A2, A3. El suceso B se puede producir en cualquiera de las tres situaciones.

Si se reescribe ahora la ecuación anterior, por ejemplo para $A 1$, tenemos

$\mathrm{P}(\mathrm{A} 1 . \mathrm{B})=\mathrm{P}(\mathrm{A} 1 / \mathrm{B}) \times \mathrm{P}(\mathrm{B})=\mathrm{P}(\mathrm{B} / \mathrm{A} 1) \times \mathrm{P}(\mathrm{A} 1)$

Aplicando álgebra elemental podemos afirmar las dos fórmulas siguientes:

1. $\mathrm{P}\left(\mathrm{A}_{1} / \mathrm{B}\right)=\left[\mathrm{P}\left(\mathrm{B} / \mathrm{A}_{1}\right) \cdot \mathrm{P}\left(\mathrm{A}_{1}\right)\right] / \mathrm{P}(\mathrm{B})$

2. $\mathrm{P}(\mathrm{B})=\mathrm{P}\left(\mathrm{B} \cdot \mathrm{A}_{1}\right)+\mathrm{P}\left(\mathrm{B} \cdot \mathrm{A}_{2}\right)+\mathrm{P}\left(\mathrm{B} \cdot \mathrm{A}_{3}\right)=\sum \mathrm{P}\left(\mathrm{B} \cdot \mathrm{A}_{\mathrm{i}}\right)=$ $\sum \mathrm{P}\left(\mathrm{B} / \mathrm{A}_{\mathrm{i}}\right) \cdot \mathrm{P}\left(\mathrm{A}_{\mathrm{i}}\right)$

Reemplazando la ecuación 2 en la ecuación 1 se obtiene el Teorema de las probabilidades condicionales enunciado por Bayes, en su Teorema, como:

$$
\mathrm{P}\left(\mathrm{A}_{1} / \mathrm{B}\right)=\left[\mathrm{P}\left(\mathrm{B} / \mathrm{A}_{1}\right) \cdot \mathrm{P}\left(\mathrm{A}_{1}\right)\right] / \sum \mathrm{P}\left(\mathrm{B} / \mathrm{A}_{\mathrm{i}}\right) \cdot \mathrm{P}\left(\mathrm{A}_{\mathrm{i}}\right)
$$

\section{Cuantificando la información a priori}

El análisis estadístico clásico, cuando por ejemplo evalúa un tratamiento frente a otro, utiliza exclusiva- mente la información obtenida en un ensayo clínico. Al contrario, en la metodología Bayesiana es fundamental el concepto de probabilidad a priori. El análisis comienza resumiendo cuantitativamente la información que existe previamente, que es externa al estudio y cuyo origen puede ser diverso, desde datos de otros estudios hasta opinión de expertos ${ }^{4,13,14}$.

Una controversia que involucra a los métodos Bayesianos se basa en la cuantificación de la distribución de probabilidad a priori, porque implica lo que podría definirse como una aparente pérdida de objetividad, ya que el análisis de toma de decisión se basa, en parte, en datos de otras realidades clínicas por fuera de la que está siendo analizada. Pero esa toma de decisiones, por ejemplo sobre eficacia de nuevos fármacos, eventos adversos o interacciones, normalmente no se fundamenta en los resultados de un único estudio ${ }^{5}$. Es muy importante aclarar que el término a priori no implica una relación temporal en el sentido de que corresponda a una información obtenida con anterioridad a nuestro estudio, sino que se refiere, en sentido más amplio, a la información externa, diferente a la de nuestro estudio ${ }^{5}$. Al haber mayor cantidad de datos disponibles de un paciente particular se podrán obtener resultados más satisfactorios. Así entonces, se trata de mejorar de manera sustancial el proceso de la toma de decisiones en base a un marco de análisis que contiene conocimiento previo relevante sobre lo que se está estudiando. Esto bien puede ser la situación de un paciente que está atravesando un enfermedad y debe ser rápida y adecuadamente tratado, y más particularmente puede también involucrar la intención de elegir el régimen farmacoterapéutico (posología) más adecuado. Esto puede ser

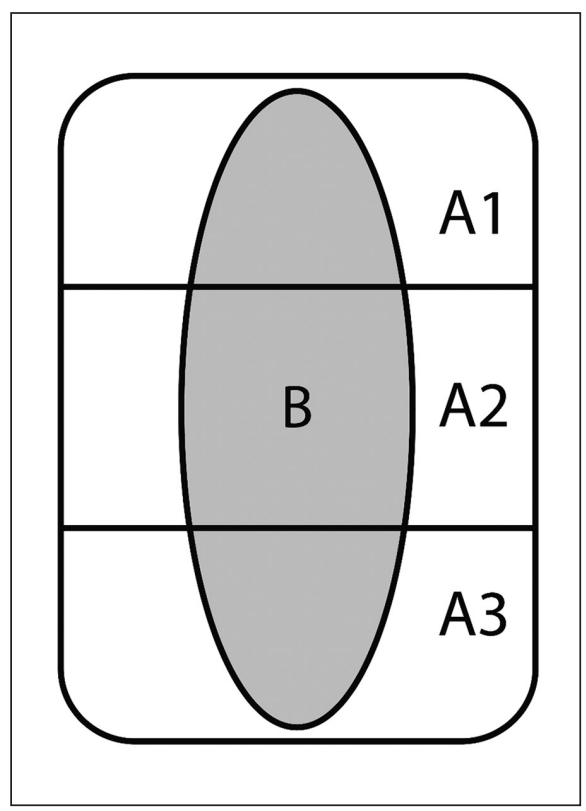

Figura 1. Representación esquemática de las probabilidades condicionales definidas por el Teorema de Bayes entre los sucesos A y $B$. 
una situación de extremo interés cuando el estado fisiopatológico del paciente depende del tratamiento de un determinado fármaco que debe ser monitorizado, por ejemplo con sus concentraciones plasmáticas ${ }^{14}$.

\section{Inferencia Bayesiana: Campos de Aplicación y Clínica}

El área de aplicación de la metodología Bayesiana es la misma que el de la estadística clásica (frecuentista). La estadística Bayesiana puede analizar diversos tipos de variables biológicas, tanto si son cuantitativas continúas o discontinuas, incluso si son cualitativas, admitiendo la posibilidad de trabajar con variables cuya distribución es normal o no-normal. Además, el enfoque Bayesiano puede aportar una renovación importante al análisis de datos ${ }^{3,4}$. Uno de los principales campos de aplicación de la inferencia Bayesiana es la investigación científica y el análisis de grandes cantidades de información (big data), lo que hoy es unas de las principales y más confiables fuentes en las que se apoyan decisiones que terminan en cambios en regímenes de tratamientos para diversas patologías y poblaciones ${ }^{11,15,16}$. En biociencias, la estadística Bayesiana también permite, por ejemplo, predecir el nivel de sensibilidad de las células cancerosas a los medicamentos basándose en datos de expresión génica de ciertos tipos de cáncer ${ }^{17}$.

Un problema importante al que se enfrenta el análisis Bayesiano es la falta de programas informáticos de fácil manejo que permitan realizar dichos abordajes. Además, en ciertas ocasiones el costo computacional requerido sobrepasa el poder de procesamiento de datos que está normalmente disponible ${ }^{13}$.

Un método, llamado Modelado Bayesiano Computacional $(\mathrm{MBC})$, fue diseñado para solucionar muchos de estos inconvenientes ${ }^{18}$. A partir de datos experimentales, la información paramétrica a priori y los diferentes modelos a cotejar, el MBC brinda la posibilidad de obtener un valor de verosimilitud paramétrica con los modelos y la distribución a posteriori que estos deben cumplir para consolidar dicho modelo. Así, deja librado al criterio del investigador aceptar o rechazar un modelo determinado en base al significado biológico o clínico del parámetro obtenido. Esto último se basa en lo que también sucede en muchos análisis de correlación de variables, en los que además de criterios gráficos y estadísticos, el significado clínico de los mismos también condiciona la lógica que determinará su inclusión, o no, en un determinado modelo final. Además, lo que corresponde a un considerable avance en el campo de la estadística Bayesiana, permite obtener un estadístico llamado "Evidencia", que posibilita la comparación entre modelos de muy distinto número de parámetros. El MBC ha sido aplicado exitosamente para investigar cómo responde el sistema inmune ante la formación del granuloma en casos de pacientes con tuberculosis ${ }^{19}$, en la interpretación de cambios conformacionales indetectables en canales iónicos activados por ligando y en el tipo de mecanismo de acción mediado por moléculas señalizadoras de daños posterior al accidente cerebro vascular (que expanden el daño a otras áreas del cerebro o predisponen a distintas regiones cerebrales a sufrir nuevos ACV), entre otros campos $^{20-22}$. Aunque las aplicaciones directas del MBC en investigaciones clínicas aún están en estadios muy iniciales, su potencial aplicación tanto en el ámbito de la infectología como en campo de lo relacionado a los procesos inflamatorios, neoplasias y patologías de origen neurológico, entre otros, lo hace parece potencialmente muy promisorio ${ }^{20-21}$. Asimismo, el $\mathrm{MBC}$ es útil como forma de caracterizar poblaciones de pacientes especiales, las que suelen ser excluidas de los ensayos clínicos controlados, tanto como de los estudios farmacocinéticos poblacionales clásicos. De no aplicarse este tipo de métodos se corre el riesgo de extrapolar erróneamente valores, por ejemplo, de parámetros farmacocinéticos de voluntarios sanos a otras subpoblaciones potencialmente muy diferentes, tales como pediátricas, geriátricos, embarazadas, o críticos, entre otras.

La estimación Bayesiana resulta importante como herramienta estadística aplicada en programas informáticos de optimización farmacoterapéutica cuando la monitorización farmacológica se basa en la interpretación farmacocinética. En el ajuste de los datos al modelo propuesto, su particularidad es la incorporación no sólo de la información experimental de una concentración (por ej. plasmática o sanguínea) en un tiempo determinado obtenida en un individuo, sino también de la información conocida a priori ${ }^{13,23}$. Esta última será la información poblacional sobre el comportamiento farmacocinético del fármaco, de ser posible en una cohorte de pacientes con características fisiopatológicas similares a las del que está siendo abordado para optimizar su terapia farmacológica ${ }^{4,13,23}$.

Así, esta población ya habrá sido adecuadamente estudiada, por ejemplo una cohorte pediátrica con una patología determinada, habiéndose estimado los valores de los parámetros farmacocinéticos poblacionales previos, así como los de su variabilidad asociada. Estas poblaciones, que podemos llamar "especiales", podrán ser niños, neonatos, ancianos, insuficientes renales, obesos, o pacientes críticos, entre otras condiciones que supongan una característica que, a priori, pudiera significar razón para generar un grupo diferenciado, con características farmacocinéticas del fármaco diferentes a las de otras poblaciones ${ }^{4,13}$.

En el campo de la investigación aplicada que busca 
resolver la forma en la que mejor se ajusta un régimen de dosificación de un fármaco, es importante destacar que los parámetros poblacionales que se implementan en un algoritmo Bayesiano son, según como los cambios de la variable independiente (explicativa) repercuten en las variables dependientes (explicada, o resultado), de tres tipos ${ }^{12,13}$.

- De efectos fijos: cuantifican tanto el comportamiento cinético medio de la población como las relaciones entre las diferentes variables fisiopatológicas y los parámetros farmacocinéticos $\left(\mathrm{P}_{\mathrm{j}}\right)$. Estos efectos son aquellos que surgen de la afectación de los parámetros farmacocinéticos por variables que son comunes a todos los individuos estudiados (edad, talla, sexo, superficie corporal, y aclaramiento de creatinina, entre otros).

- De efectos aleatorios interindividuales: cuantifican la magnitud de la variabilidad interindividual de cada uno de los parámetros farmacocinéticos del modelo al que se ajusta el fármaco $\left({ }^{2} \mathrm{P}_{\mathrm{j}}\right)$. Los modelos de efectos aleatorios suponen que los efectos de las variables explicativas son específicos a ciertos individuos, es decir que no son comunes a todos ellos (condiciones únicas y específicas de un paciente en particular).

- De efectos aleatorios residuales: cuantifican la magnitud de la variabilidad residual, principalmente la variabilidad intraindividual y el error analítico de las determinaciones que cuantifican el fármaco en un fluido biológico $\left(\sigma^{2} \mathrm{C}_{\mathrm{i}}\right)$.

Un modelo de regresión intenta explicar la relación que existe entre una variable dependiente y un conjunto de variables independientes. Un modelo de regresión lineal simple busca explicar la relación que existe entre la variable respuesta y una única variable explicativa. Así entonces vale aclarar que, en el contexto de una regresión lineal (cuando una línea recta determinada es la que mejor se ajusta a la nube de puntos para dos variables $\mathrm{X}$ e $\mathrm{Y}$,), se llaman residuos a las diferencias entre los valores de la variable dependiente observados y los valores que predecimos a partir de la recta de regresión. Por lo tanto, la variabilidad residual es la totalidad de la variabilidad cuantificada para cada uno de los sujetos estudiados ${ }^{13}$. A mayor cantidad de variables independientes que se incorporen dentro de una investigación que intenta explicar los motivos por los cuales un fármaco se comporta de determinada manera (mostrando ciertos parámetros farmacocinéticos), es esperable que el resultado (variable dependiente) sea mejor explicado. Aunque también, la cantidad de pacientes necesaria para un estudio será también tanto más grande cuanto más variables independientes sean incluidas ${ }^{13}$.

La estimación individualizada de los parámetros mediante esta técnica es similar a la empleada en regresión no lineal por mínimos cuadrados, que consiste en minimizar la suma de los cuadrados de los errores ${ }^{12,13}$. En el caso Bayesiano, la suma de cuadrados incluye un término adicional que lo hace particularmente interesante, y que evalúa las diferencias entre los parámetros estimados en el paciente, a partir de sus concentraciones séricas y los parámetros predichos, considerando la población a la que pertenece dicho paciente, adoptando entonces la siguiente ecuación:

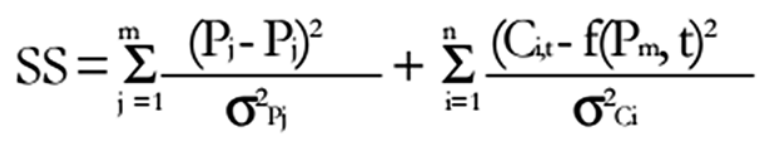

siendo $\mathrm{P}$ el número de parámetros a estimar, $\mathrm{P}_{\mathrm{i}}$ los valores medios de los parámetros farmacocinéticos poblacionales, $\mathrm{P}_{\mathrm{j}}$ los parámetros farmacocinéticos estimados en el individuo, $\sigma_{\mathrm{pj}}^{2}$ la varianza interindividual de los parámetros en la población, n el número de concentraciones conocidas en el individuo, $\mathrm{C}_{\mathrm{i}}$ los valores observados de dichas concentraciones, $f(P, t)$ los valores de concentración predichos de acuerdo al modelo cinético propuesto y $\sigma_{\mathrm{Ci}}^{2}$ la varianza residual de las concentraciones observadas ${ }^{12,13}$.

A la vista de dicha ecuación, en la que el primer término se refiere a lo poblacional y el segundo a lo individual, se deduce que cuando la información individual de concentraciones es cuantitativamente importante, el primer término de la sumatoria pierde influencia en la estimación de los parámetros y el método se asemeja a una regresión no lineal. Cuando se dispone de poca cantidad de información experimental, la influencia de los parámetros de población en el ajuste es grande, evitando así obtener valores de parámetros individuales muy diferentes a los predichos de acuerdo a sus características fisiopatológicas ${ }^{13}$.

\section{Farmacocinética aplicada}

La farmacocinética clínica, en la farmacoterapia, ha sido responsable de la expansión de la monitorización (cuantificación de un fármaco en un fluido biológico, normalmente plasma o sangre), también facilitada por desarrollos analíticos y matemático-estadísticos aplicables a la individualización posológica, como la farmacocinética poblacional y la aplicación de métodos Bayesianos $^{24}$.

Los métodos de dosificación a priori utilizan características conocidas del fármaco, así como del paciente y las patologías, que pueden influir en los parámetros farmacocinéticos ${ }^{12}$. La farmacocinética poblacional ha supuesto un notable avance, identificando y cuantificando características fisiopatológicas y de tratamiento en una población de pacientes de- 
terminada, explicando la variabilidad farmacocinética interindividual, y aumentando significativamente la capacidad predictiva. Esto permite el desarrollo de criterios posológicos específicos para poblaciones y fármacos específicos ${ }^{12}$.

De esta forma, establecer dosis que resultan más seguras y eficaces ha sido posible cuando se llevan a cabo estudios farmacocinéticos poblacionales. Entre muchos ejemplos vale destacar aquellos que, a pesar de su complejidad, se han desarrollado en ámbitos clínicos y que involucran a fármacos de estrecho margen terapéutico en grupos poblacionales de pacientes complejos. Tal el caso de la vancomicina en neonatos pretérmino o de la amikacina en niños con fibrosis quística. Ambos ejemplos muestran que la estimación de parámetros farmacocinéticos de esos antibióticos en ciertas poblaciones puede consolidarse hacia el paso siguiente, que es la sugerencia concreta de la dosis y la frecuencia que el paciente deberá recibir. Así se intenta minimizar la probabilidad de aparición de eventos adversos y aumentar la probabilidad de lograr la eficacia terapéutica deseada ${ }^{25,26}$.

A modo de ejemplo, la tabla 1 muestra valores de concentraciones de algunos antimicrobianos asociados a sus márgenes terapéuticos, así como otras consideraciones que sirven a los fines de su optimización farmacoterapéutica. Los objetivos de monitoreo que se muestran en esa tabla pueden ser mejor y más rápidamente alcanzados si se aplican los principios de la metodología Bayesiana ${ }^{27}$.

Tabla 1. Ejemplos de valores asociados al monitoreo de algunos antimicrobianos en neonatos

\begin{tabular}{|c|c|c|c|}
\hline Fármaco & Muestreo & Margen terapéutico & Observaciones \\
\hline Amikacina & $\begin{array}{l}\text { Pico: } 30 \text { min después del fin } \\
\text { de la infusión IV de } 30 \text { min } \\
\text { Valle }\end{array}$ & $\begin{array}{l}\text { Régimen de una dosis diaria: } \\
\text { Pico: } 35-64 \mu \mathrm{g} / \mathrm{mL} \\
\text { Valle: }<1 \mu \mathrm{g} / \mathrm{mL} \\
\text { Régimen de múltiples dosis diarias: } \\
\text { Pico: } 15-40 \mu \mathrm{g} / \mathrm{mL} \\
\text { Valle: } 1-8 \mu \mathrm{g} / \mathrm{mL}\end{array}$ & $\begin{array}{l}\text { Picos se correlacionan más con la eficacia y } \\
\text { valles más con la toxicidad } \\
\text { El objetivo de la relación Cmax/CIM debería } \\
\text { ser, aproximadamente, de } 8 \text { a } 10 \\
\text { Picos objetivos dependen del tipo de } \\
\text { bacteria y de infección, y de las condiciones } \\
\text { fisiopatológicas y la evolución del paciente }\end{array}$ \\
\hline Gentamicina & $\begin{array}{l}\text { Pico: } 30 \text { min después del fin } \\
\text { de la infusión IV de } 30 \text { min } \\
\text { Valle }\end{array}$ & $\begin{array}{l}\text { Régimen de una dosis diaria: } \\
\text { Pico: } 20-30 \mu \mathrm{g} / \mathrm{mL} \\
\text { Valle: }<1 \mu \mathrm{g} / \mathrm{mL} \\
\text { Régimen de múltiples dosis diarias: } \\
\text { Pico: } 4-10 \mu \mathrm{g} / \mathrm{mL} \\
\text { Valle: }<2 \mu \mathrm{g} / \mathrm{mL}\end{array}$ & Ídem Amikacina \\
\hline Tobramicina & $\begin{array}{l}\text { Pico: } 30 \text { min después del fin } \\
\text { de la infusión IV de } 30 \text { min. } \\
\text { Valle }\end{array}$ & $\begin{array}{l}\text { Régimen de una dosis diaria: } \\
\text { Pico: } 20-30 \mu \mathrm{g} / \mathrm{mL} \\
\text { Valle: }<1 \mu \mathrm{g} / \mathrm{mL} \\
\text { Régimen de múltiples dosis diarias: } \\
\text { Pico: } 4-10 \mu \mathrm{g} / \mathrm{mL} \\
\text { Valle: }<2 \mu \mathrm{g} / \mathrm{mL}\end{array}$ & Ídem Amikacina \\
\hline Vancomicina & $\begin{array}{l}\text { Valle } \\
\text { Pico: } 1 \mathrm{~h} \text { después de } \\
\text { terminada la infusión de } 1 \mathrm{~h}\end{array}$ & $\begin{array}{l}\text { Valle: } 5-20 \mu \mathrm{g} / \mathrm{mL} \\
\text { Pico: } 20-50 \mu \mathrm{g} / \mathrm{mL} \text {, solo necesario en casos } \\
\text { de bacteria caracterizada y si es posible } \\
\text { para lograr un mejor cálculo del AUC, y en } \\
\text { tratamientos de infecciones complejas } \\
\text { Infusión continua: concentración durante } \\
\text { infusión (muestra durante la infusión, no } \\
\text { valle): } 15-20 \mathrm{mg} / \mathrm{L}\end{array}$ & $\begin{array}{l}\text { Valor objetivo de AUC } 24 \mathrm{~h} / \mathrm{MIC} \text { sugerido } \\
\text { (validado en adultos): > } 400 \mathrm{mg} \mathrm{h/L}\end{array}$ \\
\hline Voriconazol & Valle & $0,5-3 \mu \mathrm{g} / \mathrm{mL}$ & $\begin{array}{l}\text { Ajustes de regímenes deben ser realizados } \\
\text { con precaución ya que el voriconazol, con } \\
\text { una eliminación hepática saturable, sigue } \\
\text { una farmacocinética no lineal }\end{array}$ \\
\hline
\end{tabular}

Valles: cuantificación del fármaco en una muestra tomada inmediatamente antes de una próxima dosis. 
En resumen, el desarrollo de un adecuado modelo farmacocinético poblacional puede ayudar a determinar una mejor posología de fármacos de dosis críticas en muchos pacientes complejos ${ }^{25}$.

Programas informáticos como $\mathrm{NONMEM}^{\circledR}$, Monolix ${ }^{\circledR}$, S-ADAPT ${ }^{\circledR}$ o Pmetrics ${ }^{\circledR}$, entre otros, sirven al modelado paramétrico poblacional, es decir que permiten estimar parámetros farmacocinéticos de un fármaco en un determinado grupo de pacientes estu$\operatorname{diados}^{13,26,28}$.

Además, un grupo de métodos denominados de control adaptado se basa en utilizar, al ajustar regímenes posológicos, los valores de parámetros farmacocinéticos obtenidos a partir de concentraciones séricas en un paciente individual. Entre ellos se encuentran los métodos Bayesianos cuya particularidad reside en una adecuada combinación de información farmacocinética, individual y poblacional $1^{4,23}$.

El uso de los métodos Bayesianos en monitorización ha flexibilizado considerablemente la selección del modelo cinético, posibilitando que estos sean llevados a la práctica de la rutina clínica ${ }^{29}$. Así por ejemplo se han podido disminuir la cantidad de muestras biológicas necesarias que deben ser analizadas, y que los tiempos de extracción no deban ser necesariamente siempre los mismos para todos los pacientes ${ }^{4}$.

Para la interpretación correcta de los resultados de concentraciones que fueron determinadas es necesario utilizar un modelo farmacocinético que intente simplificar el complejo sistema biológico del organismo, así como los procesos que experimenta el fármaco ${ }^{13}$. Dichos modelos explican el comportamiento cinético básico del fármaco mediante ecuaciones que describen la evolución de las concentraciones del fármaco en el organismo en función del tiempo ${ }^{13}$. Estas ecuaciones farmacocinéticas permiten el cálculo de los parámetros cinéticos en el individuo y la posterior individualización posológica ${ }^{29}$.

\section{Ventajas de la Estimación Bayesiana ${ }^{12,13,26,29}$}

- Información experimental mínima. Teóricamente el número necesario de muestras sanguíneas es menor que el necesario para estimar los parámetros farmacocinéticos individuales mediante regresión no lineal $(\mathrm{n}>1)$.

- Flexibilidad en los tiempos para toma de muestra de sangre. La selección de los tiempos de muestreo es menos crítica cuando se utilizan métodos Bayesianos. Es decir que, si las variables implicadas en el tratamiento (dosis, horario de administración, tiempo de infusión, volumen total administrado, y valores antropométricos del paciente, entre otros) han sido correctamente registradas, es potencial- mente posible considerar concentraciones plasmáticas, o sanguíneas, de tomas de muestras realizadas en horarios diferentes a los normalmente estipulados en las tablas clásicas de monitoreo de fármacos, y aun así simular estados de concentraciones en función del tiempo que permitan optimizar las posologías de un paciente particular.

- Versatilidad de modelos farmacocinéticos. Las técnicas Bayesianas han mostrado su utilidad no solo para modelos mono o bicompartimental, sino para otros más complejos, como por ejemplo el tricompartimental. La utilización de modelos no lineales y de concentraciones en situación de no equilibrio (que no pertenezcan al estado estacionario) pueden también ser contemplados.

- Consistencia en los resultados. La estimación Bayesiana evita obtener parámetros muy alejados del margen de variación de los parámetros poblacionales, minimizando, entre otros, los riesgos inherentes a la técnica analítica, interacciones y errores de medicación, entre otras situaciones de la rutina clínica. Proporcionan, además, recomendaciones de dosificación consistentes y confiables.

\section{Limitaciones de la Estimación Bayesiana ${ }^{12,13}$}

- Complejidad de cálculo. La aproximación Bayesiana es relativamente compleja, requiriendo de software específicos. No todos los programas informáticos que asisten en la optimización farmacoterapéutica incluyen la posibilidad de un ajuste Bayesiano.

- Fiabilidad de los parámetros de población. Una inadecuada selección de la información poblacional o su incorrecta caracterización, influyen notablemente en la fiabilidad y capacidad predictiva de los métodos Bayesianos, especialmente si la información individual es limitada. La ausencia de información poblacional limita la utilización de esta estrategia para nuevos fármacos o para poblaciones específicas y vulnerables, especialmente en pediatría, y también en otras como geriatría y pacientes críticos, entre muchos otros. Muy particularmente en estos casos, la experiencia del profesional especialista en farmacología será determinante para interpretar correctamente los datos asociados a un paciente particular.

Vale aclarar que, independientemente de las consideraciones asociadas a ventajas y limitaciones de la implementación de las metodologías Bayesianas en el campo de la farmacología clínica, existen recomendaciones directas o indirectas de organismos regulatorios de referencia a nivel mundial, tales como la FDA y la 
EMA. Esto no sucede aun en documentos similares de agencias regulatorias de Latinoamérica. Muchos documentos de la FDA y EMA sugieren la implementación de metodologías Bayesianas, o directamente la aplican, cuando se evalúa eficacia o seguridad de moléculas con actividad farmacológica, tanto en el ámbito de la industria farmacéutica y la aprobación de fármacos, como en el campo de la investigación clínica y la rutina práctica, incluyendo el campo de la pediatría.

\section{Conclusiones}

La optimización farmacoterapéutica basada en la estimación Bayesiana se aplica ampliamente en la actualidad, siendo el método de referencia para ajustes de regímenes de dosificación. Esto es particularmente conveniente y recomendable para la práctica clínica del ajuste de rutina de los regímenes de dosificación de fár- macos de estrecho margen terapéutico que optimizará importantes aspectos de la farmacoterapia del paciente. Además, suma la ventaja de requerir solo un limitado número de muestras por paciente, y la flexibilidad en cuanto a los tiempos requeridos en el muestreo de sangre para cuantificación de fármacos ${ }^{4,13,26,30}$.

El empleo de las PSE se encuentra muy arraigado en la práctica cotidiana, pese a lo cual es posible que el uso de los IC y la estadística Bayesiana continúe ganando espacio en la rutina estadística aplicada a la clínica, tanto como a la investigación básica y aplicada ${ }^{1,9,11}$. Así, la utilización de los principios Bayesianos también en la práctica de la farmacocinética clínica podrá resultar en la mejora de la atención farmacoterapéutica ${ }^{31-34}$.

\section{Conflicto de intereses}

Los autores declaran no tener conflicto de intereses.

\section{Referencias}

1. Sarria Castro M, Silva Ayçaguer LC. Tests of statistical significance in three biomedical journals: a critical review. Rev Panam Salud Publica 2004;15(5):300-6.

2. Hernández Hurtado J. Breve historia de la estadística. Maestría en Enseñanza de las Ciencias Exactas y Naturales. Universidad Nacional de Colombia. Medellín, Colombia. 2013.

3. Furst T, Strojil J. A patient called Medical Research. Biomed Pap Med Fac Univ Palacky Olomouc Czech Repub 2017;161(1):54-7.

4. Zhang T, Lipkovich I, Marchenko O. Bridging data across studies using frequentist and Bayesian estimation. J Biopharm Stat 2017;27(3):426-41.

5. Buchinsky FJ, Chadha NK. To P or Not to P: Backing Bayesian Statistics. Otolaryngol Head Neck Surg 2017;157(6):915-8.

6. Watson JV. A brief history of numbers and statistics with cytometric applications. Cytometry 2001;15;46(1):1-22.

7. Shi WL, Tang HL, Zhai SD. Effects of the CYP3A4*1B Genetic Polymorphism on the Pharmacokinetics of Tacrolimus in Adult Renal Transplant Recipients: A Meta-Analysis. PLoS One 2015;310(6):e0127995.

8. Mannocci A, Bontempi C, Giraldi G, et al. EpiInfo as a research and teaching tool in epidemiology and statistics: strengths and weaknesses. Ig SanitaPubbl 2012;68(1):8596.

9. Candia R, Caiozzi G. Confidence intervals. Rev Med Chile 2005;133(9):1111-5.

10. Cáceres Guido P. The beginning of clinical pharmacokinetics. Lat Am J Pharm 2007;26(3):462-7.

11. Pedroza C, Tyson JE, Das A, Laptook A, Bell EF, Shankaran S; Eunice Kennedy Shriver National Institute of Child Health and Human Development Neonatal Research Network. Advantages of Bayesian monitoring methods in deciding whether and when to stop a clinical trial: an example of a neonatal cooling trial. Trials. 2016;17(1):335.

12. Jiménez Torres NV, Casabo Alós VG, Chust VS. Principios farmacocinéticos básicos, en: Manual de procedimientos para farmacocinética clínica. Editorial AFAHPE "Fundación para el desarrollo clínico de la Farmacia”. Valencia, España. 1997.

13. Calvo MV, García MJ, Martínez J, Fernández MM. Farmacocinética Clínica. Sociedad Española de Farmacia Hospitalaria (SEFH). Madrid, España. 2007.

14. Ding P, Vander Weele TJ. Sensitivity Analysis Without Assumptions. Epidemiology 2016;27(3):368-77.

15. GBD 2017 Population and Fertility Collaborators. Population and fertility by age and sex for 195 countries and territories, 1950-2017: a systematic analysis for the Global Burden of Disease Study 2017. Lancet 2018;10;392(10159):1995-2051.

16. Bouckaert R, Lemey P, Dunn M, et al. Mapping the origins and expansion of the Indo-European language family. Science 2012;24;337(6097):957-60

17. Liu Q, Muglia LJ, Huang LF. Network as a Biomarker: A Novel Network-Based
Sparse Bayesian Machine for PathwayDriven Drug Response Prediction. Genes (Basel) 2019;9;10(8).

18. Fernández Do Porto DA, Auzmendi J, Peña D, García VE, Moffatt L. Bayesian approach to model CD137 signaling in human M. tuberculosis in vitro responses. PLoSOne 2013;8(2):e55987.

19. Fernández Do Porto, Darío Augusto. "La vía de CD137 regula de manera diferencial las respuestas innatas y adaptativas en el contexto de la tuberculosis humana". Tesis Doctoral, Universidad de Buenos Aires. Facultad de Ciencias Exactas y Naturales. 2013.

20. Auzmendi JA. "Desarrollo de tecnología para el estudio de la activación rápida de canales iónicos". Tesis Doctoral, Universidad de Buenos Aires. Facultad de Ciencias Exactas y Naturales. 2012.

21. Auzmendi JA, Moffatt L, Ramos AJ. Bayesian Computational Modeling: a new tool for understanding the reactive gliósis propagation. 2nd FALAN CONGRESS. FALAN-IBROLARC Buenos Aires, Argentina. 2016.

22. Auzmendi JA, Ramos AJ, Moffatt L. Translating biological mechanism into computational models of the reactive gliosis. $2^{\circ}$ Reunión Conjunta de Biociencias. Buenos Aires, 2017.

23. Marks L, Duchein S, González II, Suárez HA, Rivolta S. Validation of a immunoassay for the determination of vancomycin in cerebospinal fluid. Rev Fac Cien Med Univ Nac Cordoba 2019;19;76(2):101-6.

24. Sheiner LB, Rosenberg B, Marathe VV. Estimation of population characteristics 
of pharmacokinetic parameters from routine clinical data. J Pharmacokinet Biopharm 1977;5(5):445-79.

25. Cáceres Guido P, Travaglianti M, Castro G, et al. Population Pharmacokinetic Study of Vancomycin in Preterm Neonates. Lat. Am. J. Pharm 2015;34(1):124-33.

26. Cáceres Guido P, Pérez M, Halac A, et al. Population pharmacokinetics of amikacin in patients with pediatric cystic fibrosis. Pediatric Pulmonology 2019;35(0);1293310.

27. Cáceres Guido P. Monitoreo terapéutico de fármacos en neonatología. En: Travaglianti M. Vademecum Neonatal 2019: Guía farmacoterapéutica para el recién nacido y los primeros meses de vida. Provincia de Buenos Aires, Argentina. Edifarma. 2019;327-30.

28. Woillard JB, Debord J, Monchaud C, Saint-Marcoux F, Marquet P. Population Pharmacokinetics and Bayesian Estimators for Refined Dose Adjustment of a New Tacrolimus Formulation in Kidney and Liver Transplant Patients. Clin Pharmacokinet 2017;56(12):1491-8.

29. Basu C, Ahmed MA, Kartha RV, et al. A hierarchical Bayesian approach for combining pharmacokinetic/ pharmacodynamic modeling and Phase IIa trial design in orphan drugs: Treating adrenoleukodystrophy with Lorenzo's oil. J Biopharm Stat 2016;26(6):1025-39.
30. Becker P, Besa S, Riveros S, et al. Results of a national program of pediatric heart transplantation: strengths and weakness. Rev Chil Pediatr 2017;88(3):367-76.

31. FDA (Food and Drug AdministrationUS). Population Pharmacokinetics, Guidance for Industry. Population Pharmacokinetics; Revised Draft Guidance for Industry; Availability. FDA-2019-D-2398 U.S. Office of Medical Products and Tobacco, Center for Drug Evaluation and Research - 2019. Available at: https://www.federalregister.gov/ documents/2019/07/12/2019-14856/ population-pharmacokinetics-reviseddraft-guidance-for-industry-availability. Accessed March 13, 2020. 